\title{
I. NOMENCLATURE
}

\section{Generalized Linear Solution of Proportional Navigation}

\author{
U.S. SHUKLA \\ P.R. MAHAPATRA
}

Indian Institute of Science

Proportional navigation (PN) equations are not solvable in closed form. Linearized solutions have been widely used for PN system analysis and design, but these are based on overly restrictive assumptions regarding the initial geometry, and are valid only for near-tail-chase pursuits. A generalization of the linearized approach is presented which yields more accurate estimates of pursuer lateral acceleration than the classical linear solutions as verified by comparison with "exact" numerical solutions. Further, the solution is applicable over a much wider range of engagement geometries. The treatment is based on a closed-form quasilinearized solution of the PN equations followed by the small-angle approximation only to line of sight (LOS) angle rate.

Manuscript received March 10, 1987; revised October 2, 1987.

Authors' address: Indian Institute of Science, Department of Aerospace Engineering. Bangalore 560012, India.

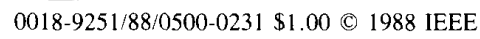

Variables

$A \quad$ Lateral acceleration (normal to velocity vector).

$N \quad$ Navigation constant.

$V \quad$ Velocity.

$g \quad$ Acceleration due to gravity.

$r \quad$ Range from pursuer to target.

$t$ Time.

$\phi \quad$ Angle between pursuer velocity vector and reference line.

$\Delta \phi$ Heading error relative to collision course.

$\theta$ Angle of line of sight relative to reference line.

Subscripts

CL Classical linear.

GL Generalized linear.

$M$ Pursuer.

QL Quasilinear,

$T$ Target.

$i \quad$ Initial values.

$f \quad$ Final value (at intercept).

$n$ Iteration number.

0 Zeroth order (initial) approximation.

c (for) Constant bearing course.

rc Constant relative (velocity) along line of sight.

ri Initial relative (velocity).

riL Initial relative (velocity) along line of sight.

\section{INTRODUCTION}

Proportional navigation (PN) is a widely used guidance law for short-range homing and interception. However, the equations governing PN are highly nonlinear even in their simplest form where the target and the pursuer are considered to be inertialess points.

General closed-form solutions to these equations have not been reported even for the "simple" case of nonmaneuvering target motion with constant target and pursuer speeds, except under highly restrictive conditions [1-3], though some qualitative insight has been generated for somewhat more general situations $[4,5]$.

In the absence of closed-form solution to the PN equations, attention in the past has been primarily focused on linearized solutions [3]. Such solutions are reasonably accurate when the angles involved in the geometry are small. Because of their closed-form nature, linear solutions provide good insight into the PN system behavior and are extensively used for off-line calculation for design and analysis of systems involving the PN strategy. However, the currently used linear solutions are severely limited in accuracy when the geometry differs significantly from a constant-bearing or collision situation.

A more general form of linearized solution to the geometric PN equations has been derived, which provides more accurate results than the classical linear (CL) approach. Further, comparison with "exact" numerical solution shows that the generalized linear (GL) solution is 
valid for large departures of the geometry from a collision course - a case for which the CL solution is not applicable.

The approach taken to derive the GL solution is to first obtain a closed-form quasilinearized (QL) solution of the PN equations and then reduce it to a linear solution through suitable approximations.

\section{QUASILINEARIZED SOLUTION OF THE PROPORTIONAL NAVIGATION EQUATIONS}

Considering the PN geometry of Fig. 1 for a nonmaneuvering target, the equations describing the

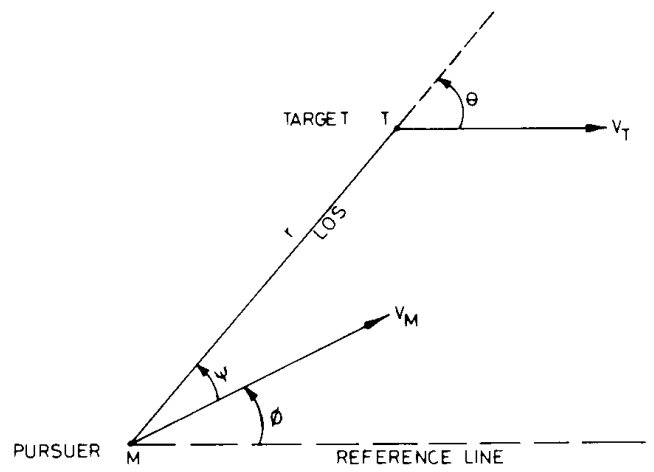

Fig. 1. Geometry of PN

motion of the pursuer may be written as [2]

$\dot{r}=V_{T} \cos \theta-V_{M} \cos (\theta-\phi)$

and

$r \dot{\theta}=-V_{T} \sin \theta+V_{M} \sin (\theta-\phi)$.

The definition of PN

$\dot{\phi}=N \dot{\theta}$

can be integrated to give

$\phi=\phi_{i}+N\left(\theta-\theta_{i}\right)$

which can be rearranged as

$\theta-\phi=b \theta-c$

where $b=1-N$ and $c=\phi_{i}-N \theta_{i}$.

Using (5) in (1) and (2) we get

$\dot{r}=V_{T} \cos \theta-V_{M} \cos (b \theta-c)$

and

$r \dot{\theta}=-V_{T} \sin \theta+V_{M} \sin (b \theta-c)$.

If range-to-go $r$ is a monotonic function of time $t$, as is the case with the normal range of PN parameters, (6) and (7) can be rewritten with $r$ as the independent variable:

(2) $\frac{d t}{d r}=\frac{1}{V_{T} \cos \theta-V_{M} \cos (b \theta-c)} \stackrel{\triangleq}{=} d(\theta)$

and

$r \frac{d \theta}{d r}=\frac{-V_{T} \sin \theta+V_{M} \sin (b \theta-c)}{V_{T} \cos \theta-V_{M} \cos (b \theta-c)} \triangleq g(\theta)$.

The nonlinear differential equations (8) and (9) are not solvable in closed form. We obtain an approximate analytical solution using the QL technique [6]. We begin the QL process by expanding the right-hand side (RHS) of (9) in a Taylor series in the function space and truncating the series after the first derivative term.

$r \frac{d \theta_{n}}{d r}=g_{n-1}+h_{n-1}\left(\theta_{n}-\theta_{n-1}\right)$

where

$h_{n-1}=\left.h\left(\theta_{n-1}\right) \triangleq \frac{\partial g}{\partial \theta}\right|_{(n-1)}$

$g_{n-1} \triangleq g\left(\theta_{n-1}\right)$

and $n$ denotes the iteration number. Similarly, we obtain from (8)

$\frac{d t_{n}}{d r}=d_{n-1}+e_{n-1}\left(\theta_{n}-\theta_{n-1}\right)$

where

$e_{n-1}=\left.e\left(\theta_{n-1}\right) \triangleq \frac{\partial d}{\partial \theta}\right|_{(n-1)}$

$d_{n-1} \triangleq d\left(\theta_{n-1}\right)$.

From definition, and after the necessary simplification, $h$ and $e$ are obtained as

$h \triangleq \frac{\partial g}{\partial \theta}=\frac{-b V_{M}^{2}-V_{T}^{2}+(1+b) V_{T} V_{M} \cos (N \theta+c)}{\left[V_{T} \cos \theta-V_{M} \cos (b \theta-c)\right]^{2}}$

and

(4)

$e \triangleq \frac{\partial d}{\partial \theta}=-\frac{-V_{T} \sin \theta+b V_{M} \sin (b \theta-c)}{\left[V_{T} \cos \theta-V_{M} \cos (b \theta-c)\right]^{2}}$.

The QL procedure requires that an initial approximation $\theta_{0}(r)$ to the solution (corresponding to $n=0$ ) be assumed. Subsequent approximations may then be obtained by recursive relations such as (10) and (11).

(6) Any suitable initial approximation may be used, but choosing $\theta_{0}(r)$ equal to the initial LOS angle $\theta_{i}$, i.e.,

$\theta_{0}(r)=\theta_{i}$

(7)

results in considerable simplification of the analytical expressions. Using (14) in (8), (9), (12), and (13) and further using (6) and (7), the following constants are defined 


$$
\begin{aligned}
& G_{0} \triangleq g\left(\theta_{0}\right)=\frac{-V_{T} \sin \theta_{i}+V_{M} \sin \left(b \theta_{i}-c\right)}{V_{T} \cos \theta_{i}-V_{M} \cos \left(b \theta_{i}-c\right)} \\
& =-\frac{r_{i} \dot{\theta}_{i}}{V_{\text {riL }}} \\
& H_{0} \triangleq h\left(\theta_{0}\right) \\
& =\frac{-b V_{M}^{2}-V_{T}^{2}+(1+b) V_{T} V_{M} \cos \left(N \theta_{i}+c\right)}{\left\{V_{T} \cos \theta_{i}-V_{M} \cos \left(b \theta_{i}-c\right)\right\}^{2}} \\
& =-\frac{V_{r i}^{2}}{V_{r i L}^{2}}+N^{\prime} \frac{V_{M}-V_{T} \cos \phi_{i}}{V_{r i L} \cos \phi_{c}} \\
& D_{0} \triangleq d\left(\theta_{0}\right)=\frac{1}{V_{T} \cos \theta_{i}-V_{M} \cos \left(b \theta_{i}-c\right)} \\
& =-\frac{1}{V_{r i L}} \\
& E_{0} \stackrel{\triangleq}{=} e\left(\theta_{0}\right)=-\frac{-V_{T} \sin \theta_{i}+b V_{M} \sin \left(b \theta_{i}-c\right)}{\left\{V_{T} \cos \theta_{i}-V_{M} \cos \left(b \theta_{i}-c\right)\right\}^{2}} \\
& =-\frac{r_{i} \dot{\theta}_{i}-N V_{M} \sin \left(\theta_{i}-\phi_{i}\right)}{V_{r i L}^{2}}
\end{aligned}
$$

where $V_{r L}=-\dot{r}$ is the relative closing velocity along LOS and $V_{r i L}$ is its initial value. The initial relative velocity between the target and the pursuer is $V_{r i}$.

Substituting the four constants (15)-(18) in (10) and (11), the following constant-coefficient linear differential equations are obtained for $n=1$

$r \frac{d \theta_{1}}{d r}-H_{0} \theta_{1}=G_{0}-H_{0} \theta_{i}$

and

$\frac{d t_{1}}{d r}=D_{0}+E_{0}\left(\theta_{1}-\theta_{i}\right)$.

Equations (19) and (20) are subject to the initial conditions $\theta_{1}\left(r_{i}\right)=\theta_{i}$ and $t_{1}\left(r_{i}\right)=0$.

From (19), the solution for $\theta_{1}$ is obtained as

$\theta_{1}=\theta_{i}+\frac{G_{0}}{H_{0}}\left(\left(\frac{r}{r_{i}}\right)^{H_{0}}-1\right)$.

Substituting from (21) in (20)

$$
\begin{aligned}
t_{1}= & r_{i}\left[\left(D_{0}-\frac{E_{0} G_{0}}{H_{0}}\right)\left(\frac{r}{r_{i}}-1\right)\right. \\
& \left.+\frac{E_{0} G_{0}}{H_{0}\left(H_{0}+1\right)}\left(\left(\frac{r}{r_{i}}\right)^{H_{0}+1}-1\right)\right] .
\end{aligned}
$$

The pursuer lateral acceleration $A_{M}$, normal to the pursuer velocity vector, is given by

$$
\begin{aligned}
A_{M} & \left.=\left(V_{M} / g\right) d \phi / d t \quad \text { (in } g s\right) \\
& =\left(V_{M} / g\right) N d \theta / d t, \quad \text { using (3) }
\end{aligned}
$$

$$
=\left(V_{M} / g\right) N(d \theta / d r) /(d t / d r) .
$$

Substituting the values of $d \theta_{1} / d r$ and $d t_{1} / d r$, obtained by differentiation of (21) and (22), for $d \theta / d r$ and $d t / d r$, respectively, in (23),

$A_{M}=\frac{N V_{M}}{g r_{i}}\left[\frac{G_{0}\left(\frac{r}{r_{i}}\right)^{H_{0}-1}}{D_{0}+\frac{E_{0} G_{0}}{H_{0}}\left(\left(\frac{r}{r_{i}}\right)^{H_{0}}-1\right)}\right]$.

Using the last results of (15) and (17),

$A_{M}=\frac{N V_{M}}{g r_{i}}\left[\frac{-\frac{r_{i} \dot{\theta}_{i}}{V_{r i L}}\left(\frac{r}{r_{i}}\right)^{H_{0}-1}}{-\frac{1}{V_{r i L}}+\frac{E_{0} G_{0}}{H_{0}}\left(\left(\frac{r}{r_{i}}\right)^{H_{0}}-1\right)}\right]$.

From (20) and (21)

$\frac{E_{0} G_{0}}{H_{0}}\left(\left(\frac{r}{r_{i}}\right)^{H_{0}}-1\right)=\frac{d t}{d r}-D_{0}=-\frac{1}{V_{r L}}+\frac{1}{V_{r i L}}$.

The second equality in (26) uses (17) and the definition of $V_{r L}$. Using (26) in (25)

$A_{M}=\frac{N V_{M} V_{r L}}{g r_{i}} \frac{r_{i} \dot{\theta}_{i}}{V_{r i L}}\left(\frac{r}{r_{i}}\right)^{H_{0}-1}$.

\section{GENERALIZED LINEAR SOLUTION FOR LATERAL ACCELERATION}

The QL-derived expression (27) for lateral acceleration $A_{M}$ can be cast in a form explicitly dependent on the heading error $\Delta \phi_{i}$ relative to the collision course based on the initial geometry. For this, we use the relation [7]

$\dot{\theta}_{i}=-\frac{V_{M} \cos \phi_{c}}{r_{i}} \Delta \phi_{i}$

which is valid for small values of $\theta_{i}$ and $\Delta \phi_{i}$. Then, from (27)

$A_{M}=-\frac{V_{M}}{g} \frac{V_{r L}}{r_{i}} N^{\prime}\left(\frac{r}{r_{i}}\right)^{H_{0}-1} \Delta \phi_{i}$

which we call the GL form of $A_{M}$. Equation (29) is in a form similar to the CL form [3]

$$
\begin{aligned}
A_{M} & =-\frac{V_{M}}{g t_{f}} N^{\prime}\left(1-\frac{t}{t_{f}}\right)^{N^{\prime}-2} \Delta \phi_{i} \\
& =-\frac{V_{M}}{g} \frac{V_{r^{\prime}}}{r_{i}} N^{\prime}\left(\frac{r}{r_{i}}\right)^{N^{\prime}-2} \Delta \phi_{i} .
\end{aligned}
$$

The second equality is true, since $t_{f}=r_{i} / V_{r c}$ and $t=$ $\left(r_{i}-r\right) / V_{r}$. 
The fact that (29) is a more generalized form of (30) is apparent from a structural comparison of these two expressions for $A_{M}$. The parameter $H_{0}$ in the GL solution (29) is the analog of $N^{\prime}$ in the CL solution (30) with a fixed difference of unity. This is made clear by applying small-angle approximations to the last result in (16), as shown below.

When $\theta_{i}$ is small, the heading angle $\phi_{c}$ corresponding to the collision course is also small for normal values of $V_{M} / V_{T}$. Further, for small heading errors $\Delta \phi_{i}, \phi_{i}$ would also be small. Then, from the last expression of (16),

$H_{0}=-\frac{V_{r i}^{2}}{V_{r i L}^{2}}+N^{\prime} \frac{V_{r i}}{V_{r i L}}$.

Since $V_{r i L}$ and $r_{i} \dot{\theta}_{i}$ are orthogonal, being the components of the relative initial velocity $V_{r i}$ along and perpendicular to the LOS, we have

$V_{r i L}^{2}+r_{i}^{2} \dot{\theta}_{i}^{2}=V_{r i}^{2}$

Using the expression (28)

$\frac{V_{r i}^{2}}{V_{r i L}^{2}}=1+\left(\frac{r_{i}}{\dot{r}_{i}}\right)^{2} \frac{V_{M}^{2} \cos \phi_{c}}{r_{i}^{2}}\left(\Delta \phi_{i}\right)^{2}$

$\simeq 1$ (neglecting second-order terms in $\Delta \phi_{i}$ ).

Then, using (32b) in (31)

$H_{0}=N^{\prime}-1$

This proves that the quantity $H_{0}+1$ of the GL

formulation is a generalized replacement for $N^{\prime}$ in the $\mathrm{CL}$ formulation.
Indeed, the generalization involved in GL over CL is a powerful one, in the sense of accommodating a much wider range of pursuit geometries. The linearization assumptions are only weakly used for the GL development, essentially through the LOS rate. In particular, CL requires that the constant-bearing angle $\phi_{c}$ itself be small, in addition to the launch geometry being close to the constant-bearing. In contrast, GL only requires relative closeness between the launch geometry and a constant-bearing geometry, without much restriction on $\phi_{c}$ itself. This fact is also borne out by numerical results which form the next stage of comparison between the two linearized formulations.

The lateral acceleration $A_{M}$ obtained from the GL expression (29) are plotted in Fig. 2. As a reference, for determining the absolute accuracy of the GL results, Fig. 2 also shows the value of $A_{M}$ obtained by an accurate numerical solution of the original geometric equations (1) and (2), followed by the use of (3) and (23). This numerical solution is referred to as the "exact" solution in the paragraphs below. Further, to put the GL results in perspective with those from $\mathrm{CL}$ solutions, estimates of $A_{M}$ obtained from the CL formula (30) are also included in Fig. 2.

In Fig. 2, the commonly used effective navigation constant $N^{\prime}$ values of 2,3 , and 4 are considered. Two values $\left(10^{\circ}\right.$ and $\left.60^{\circ}\right)$ are considered for the launch angle $\theta_{i}$; the first is chosen to represent the near-tail-chase condition and the second represents a large departure from it. The heading error is kept constant at $15^{\circ}$.

It is apparent from the graphs of Fig. 2 that for

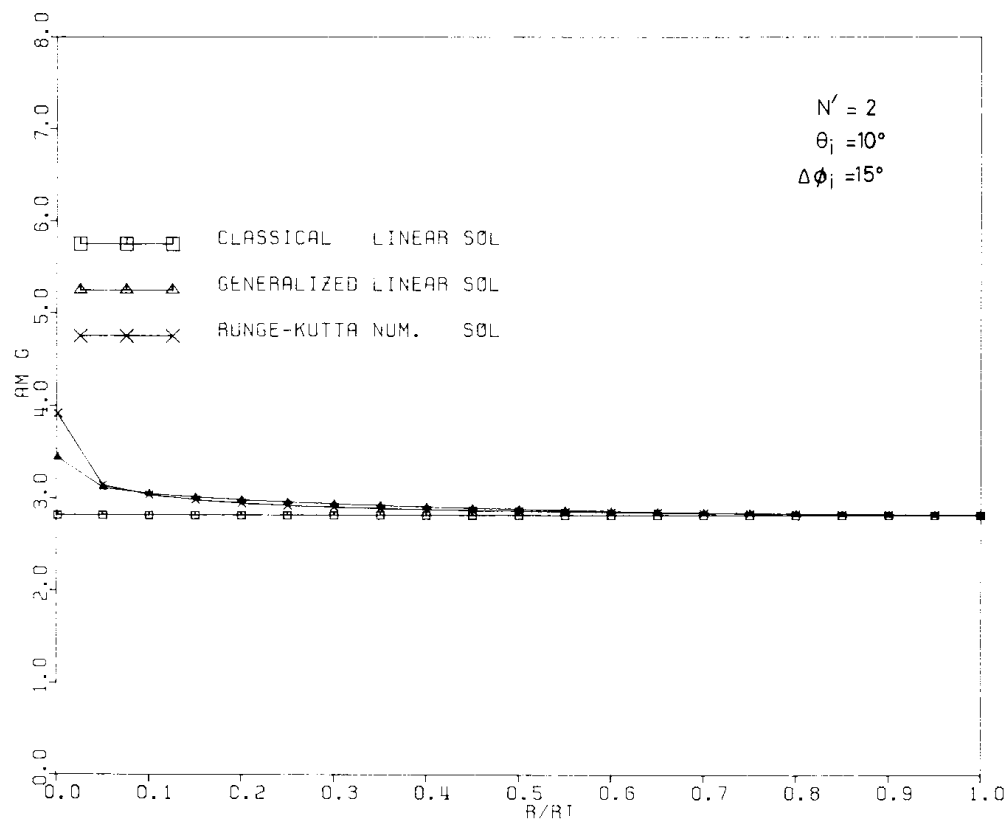

Fig. 2. Lateral acceleration as a function of normalized range. (a). (See also Fig. 2(b)-(f).) 


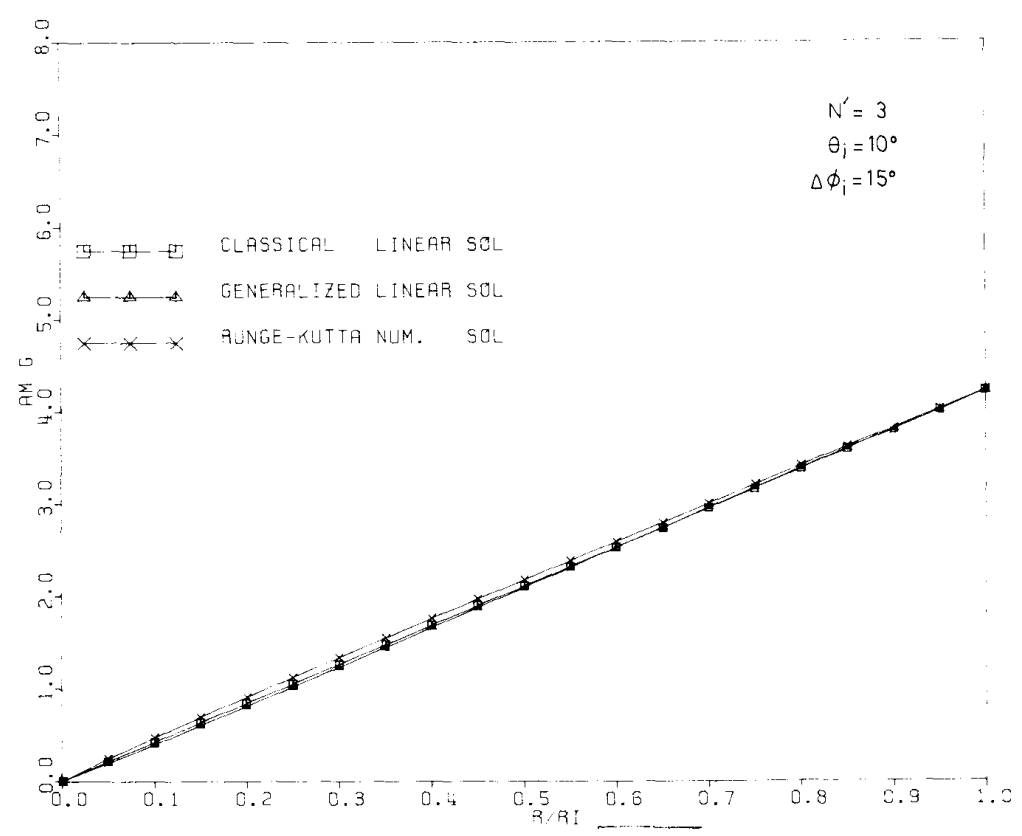

Fig. 2(b).

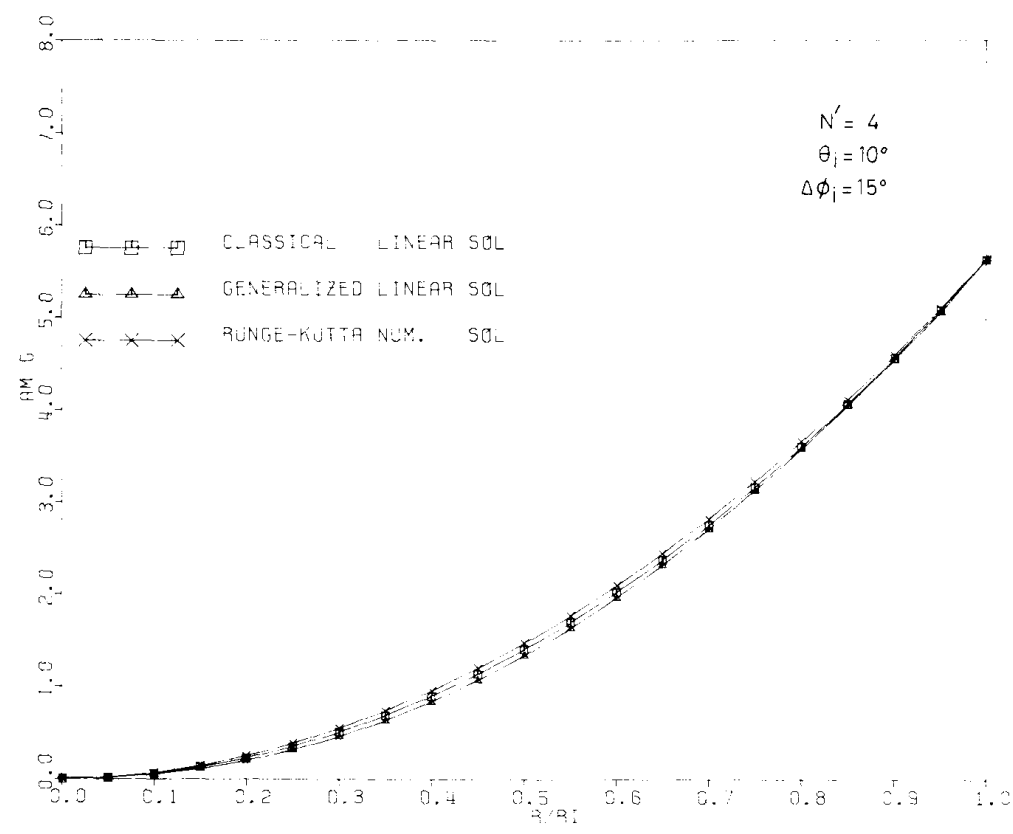

Fig. 2(c).

shallow geometries, where the pursuit remains nearly a tail-chase throughout, the $\mathrm{CL}$ results are quite close to the exact solution, and the GL results fall between the two. Thus, the GL method produces a marginal improvement in cases where the CL solution is valid. However, for steeper geometries (i.e., larger launch angles), where the CL method is no more valid, the GL method continues to provide useful estimates of the lateral acceleration $A_{M}$. The results of $\mathrm{CL}$ formulation have been included in the last three graphs of Fig. 2 to emphasize this fact, although CL results are not expected to be valid for large launch angles. The GL estimate is especially accurate in the later part of the pursuit, as the normalized distance $r / r_{i}$ goes to zero. 


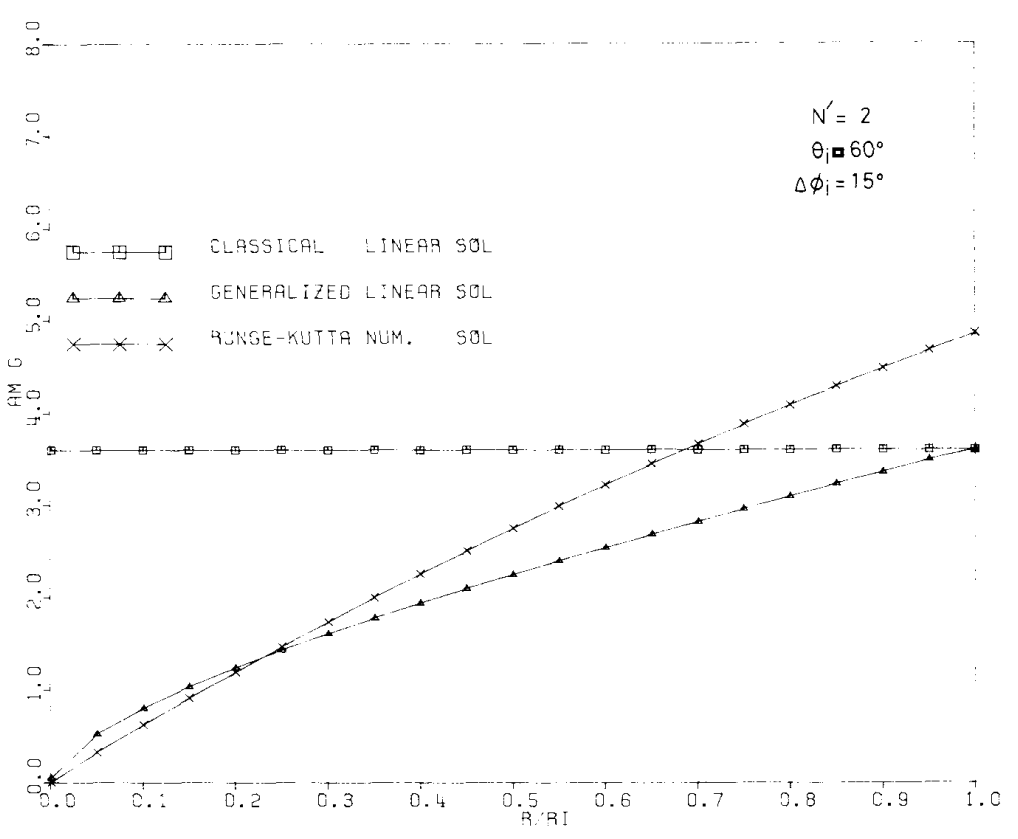

Fig. 2(d)

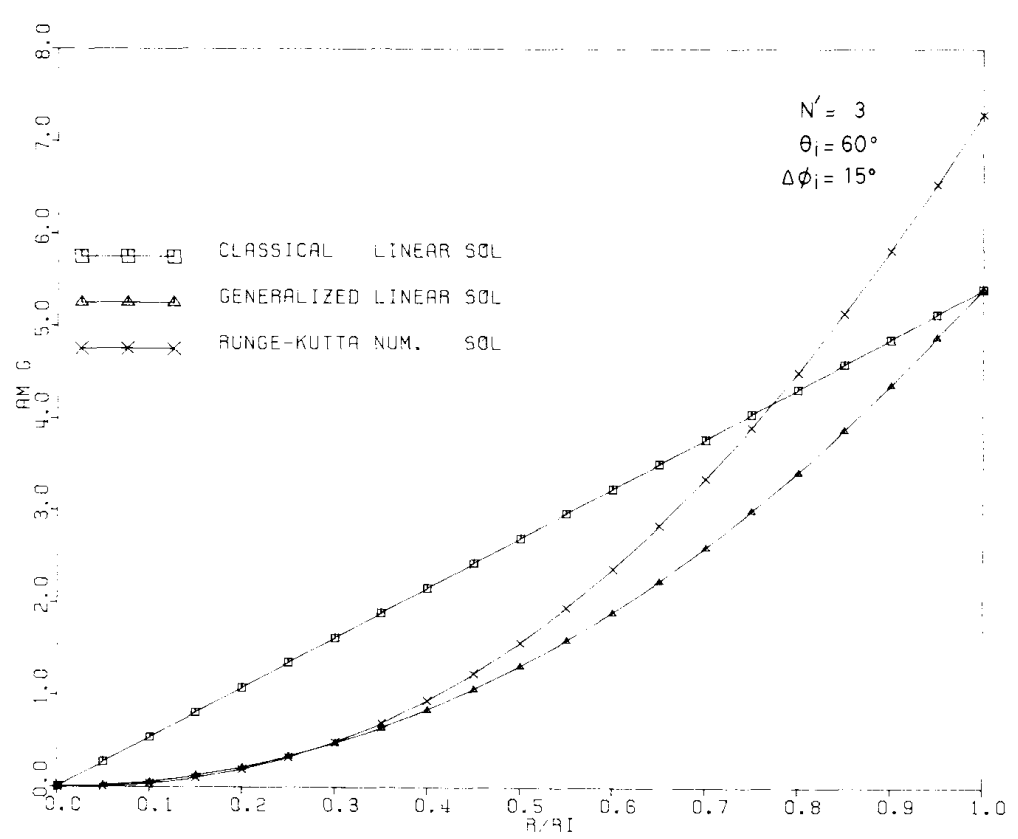

Fig. 2(e).

\section{CONCLUDING REMARKS}

A refined treatment of the proportional navigation problem within the linear framework has been the aim of this paper. First, a QL solution of the PN geometric equations has been obtained in closed form. This is further reduced to a linear form through small-angle approximations which are less restrictive than those in the $\mathrm{CL}$ approach. Specifically, for the linearization in this paper, the launch geometry is only required to be relatively close to the constant-bearing geometry, without putting severe restrictions on the constant-bearing geometry itself.

The GL approach, as it is called here, is first shown 


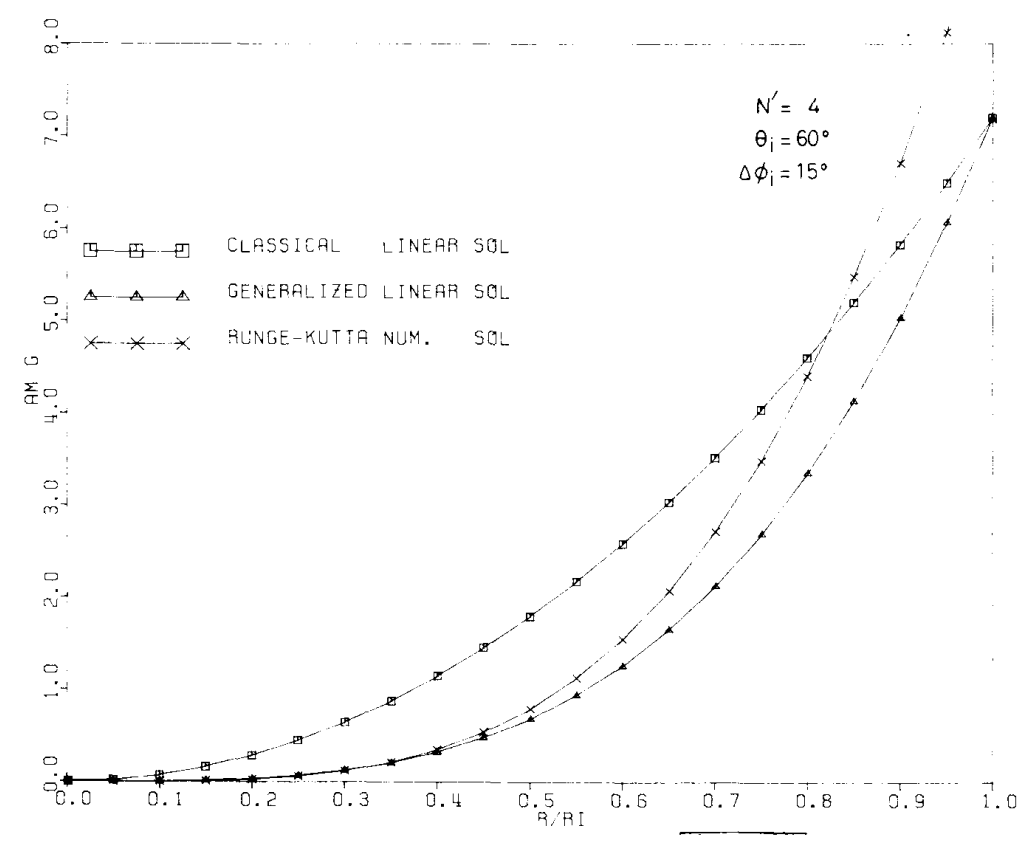

Fig. 2(f).

to be indeed a generalization of the CL formulation, and is then shown, through actual evaluation, to yield accurate estimates of the trajectory parameters. The accuracy has been established by comparison with exact numerical solutions of the original PN equations. Results from classical linear solutions have also been provided congside, only to illustrate that the GL solution is valid even for large departures from a constant-bearing geometry, where the CL treatment is not (and is indeed not expected to be) valid.

The QL approach provides options for various levels of formulation in terms of complexity and accuracy. The form presented here is considered to be a good compromise between simplicity and accuracy for a wide range of tactical situations. 


\section{REFERENCES}

[1] Guelman, M. (1976)

The closed form solution of true proportional navigation. IEEE Transactions on Aerospace and Electronic Systems AES-12 (July 1976), 472-482.

[2] Locke, A.S. (1960)

Guidance.

Princeton, N.J: Van Nostrand, 1955.

[3] Jerger, J.J. (1960)

Systems Preliminary Design

Princeton, N.J.: Van Nostrand, 1960

[4] Murtaugh, S.A., and Criel, H.E. (1966)
Fundamentals of proportional navigation.

IEEE Spectrum, 3 (Dec. 1966), 75-85.

[5] Guelman, M. (1971)

A qualitative study of proportional navigation

IEEE Transactions on Aerospace and Electronic Systems, AES-7 (July 1971), 637-643.

Bellman, R., and Kalaba, R. (1965)

Quasilinearization and Nonlinear Boundary Value Problems. New York: American Elsevier, 1965.

[7] Shinar, J. (1973)

Homing of a rolling missile against a maneuvering target. Israel Journal of Technology, 11, 3 (1973), 117-130.
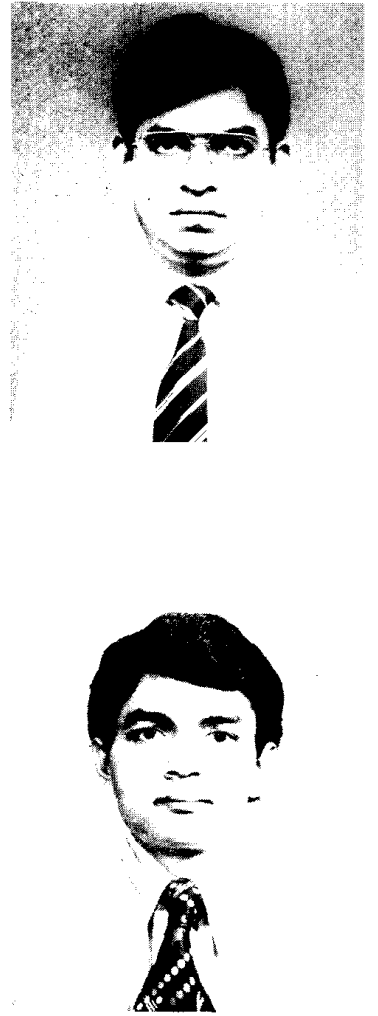

238
Uday S. Shukla was born in 1951 in Matura (U.P.), India. After receiving his Bachelor's degree in electrical communication engineering in 1973 from the Indian Institute of Science, Bangalore he has been working with Hindustan Aeronautics Limited in the same city. His areas of interest lie in the broad field of avionic systems, and navigation theory and software. Currently, Mr. Shukla is an external registrant for the Ph.D. program at the Indian Institute of Science.

Pravas R. Mahapatra received his B.Sc. (Engg) from Regional Engineering College at Rourkela, and M.E. and Ph.D. degrees from the Indian Institute of Science at Bangalore, India. He has been teaching at the Department of Aerospace Engineering in the Indian Institute of Science since 1970, where he is currently an Associate Professor.

Dr. Mahapatra has a broad area of active interest within the field of aerospace and electronic systems. This includes radar systems, navigational aids, navigation theory and flight safety aspects with particular reference to weather phenomena and air traffic control. 